\title{
The Clinic Analysis of Diclofenac Suppository for Oocyte Retrieval Analgesia in IVF-ET Cycles
}

\author{
Yubin Li, Qingyun Mai, Tao Li, Yiping Zhong, Canquan Zhou
}

The Reproductive Center, The First Affiliated Hospital of Sun Yat-sen University, Guangzhou, China. Email: liyubin97200@163.com

Received June $3^{\text {rd }}$, 2013; revised June $23^{\text {rd }}$, 2013; accepted July $11^{\text {th }}, 2013$

Copyright (C 2013 Yubin Li et al. This is an open access article distributed under the Creative Commons Attribution License, which permits unrestricted use, distribution, and reproduction in any medium, provided the original work is properly cited.

\begin{abstract}
Objective: To study the effect of diclofenac suppository in oocyte retrieval of IVF-ET. Study Design: 1176 patients with informed consents were enrolled into this prospective randomized controlled study. The setting was an IVF-ET program at the First Affiliated Hospital of Sun Yat-sen University, Guangzhou, China. According to the analgesic drug use, the patients were randomly divided into pethidine group (573 cases) and diclofenac suppository group (603 cases). The data of vital signs, common adverse reactions, severe adverse events and pain degree in oocyte retrieval were collected. The IVF-ET outcomes were also compared. Results: The post-operation pressure and pulse were lower in pethidine group than in diclofenac suppository group (both $P<0.001$ ). The rest vital signs were not statistically different (all $P>0.05$ ). Common adverse reactions in diclofenac suppository group were relatively less (all $P<0.05$ ). Pain degree between the two groups was not statistically different $(P=0.304)$. IVF-ET outcomes were also not statistically different (all $P>0.05$ ). There were 3 cases serious abdominal bleeding with shock in the diclofenac suppository group. Conclusion: Using diclofenac suppository in oocyte retrieval analgesic had a good effect. And there was no adverse effect in the IVF-ET outcome. But we should pay close attention to the probability of serious abdominal bleeding.
\end{abstract}

Keywords: Diclofenac Suppository; IVF-ET; Oocyte Retrieval; Analgesia

\section{Introduction}

With the vigorous development of assisted reproductive technology, in vitro fertilization-embryo transfer (IVFET) technique has gradually become the routine method in current infertility treatment. Oocyte retrieval in IVFET protocol is an invasive step, which is often mediated by transvaginal ultrasonography. The surgeries often lead to lower abdominal pain. And sometimes it is even unbearable. In many IVF reproductive centers, pethidine is chosen for analgesia in the operation. Other drugs such as propofol, nitrous oxide also achieved good results. But the unknown influences of narcotic drugs on the oocytes often limit the use of effective analgesic drugs. So the pain in some patients has not been effectively alleviated [1]. Diclofenac suppository had been efficiently used in gynecological and obstetric operations [2,3]. Recently in some IVF centers, it also was used in oocyte retrieval. In order to learn more about the clinical efficacy and safety of this drug in IVF treatment protocols, we carried out this clinical analysis.

\section{Materials and Methods}

\subsection{Clinical Characteristics}

1176 patients undergoing IVF treatment in our hospital from November 2011 to April 2012 were collected into the study, which included conventional IVF-ET and intracytoplasmic sperm injection (ICSI-ET). Before operations all patients signed informed consents. The study obtained the approval from the ethics committee of The First Affiliated Hospital of Sun Yat-sen University. Ovarian hyperstimulation protocols were conventional long protocols and short protocols. The patients, aged from 22 to 42 years old, didn't have any contraindications of oocyte retrieval and using of pethidine, diazepam and diclofenac in accordance with the medication instructions. They didn't have vital organs dysfunction, and didn't use aspirin and other anti-clotting drugs. Bilateral ovarian reserves of all patients were good, and the total antral follicles were more than 5 . In accordance with the analgesic drugs used before operations, the patients were randomly divided into two groups: the observation group using 
diclofenac suppository (603 cases) (Tongyao, Wuhan, China), and the control group using pethidin (573 cases) (Renfu, Yichang, China). The patients' age, body mass index (BMI), constituent ratios of ovarian hyper-stimulation protocols and insemination methods were all not statistically different compared between the two groups (all $P>0.05$ ).

\subsection{Oocyte Retrieval and the Analgesic Drugs Use}

All patients undergoing oocyte retrievals were after ovarian hyperstimulation protocols. Before operations, the patients were routinely asked common physical condition, checked vital signs and identity information. Analgesic drugs were used 10 - 15 minutes before oocyte retrieval by our nurses randomly. The patients in control group were intramuscular injected $50 \mathrm{mg}$ pethidine for analgesia. And in observation group the patients inserted $50 \mathrm{mg}$ diclofenac suppositories into rectum. The doctors had adequate oocyte retrieval experience. The operations were guided by transvaginal ultrasonography using $16 \mathrm{G}$ oocyte-retrieval needles under negative pressure (110 - 120 mmHg) on both ovaries. We must avoid the puncture needles injuring blood vessels and passing through uteruses and bladders. During the operations, we recorded the pain response, vital signs and common adverse reactions. The common adverse reactions included dizziness, palpitations, nausea, vomiting and cold sweat, etc. The nurses recorded the patients' blood pressure, pulses, oxygen saturation when finishing aspirating one side of ovary. And then the doctors continued to the other sides. When finishing oocyte retrieval, we should observe whether there was apparent abdominal active hemorrhage by ultrasound carefully. Also we must pay attention to the bleeding of vaginal wall. Finally we recorded the postoperative blood pressure, pulse, blood oxygen saturation. Oocyte retrieval time of all cases was within 15 minutes.

\subsection{Recording Severe Adverse Events}

Abdominal massive hemorrhage with shock (which needed surgery to stop bleeding), cardiovascular and cerebrovascular accidents affecting the lives were recorded.

\subsection{Evaluation Criteria of Pain Degree in Oocyte Retrieval}

In according to the patients' pain response in oocyte retrieval, it was divided into 4 grades. Grade 0: no pain, a little discomfort; Grade 1: mild pain, do not feel abdominal pain when distracted, only feel brief tingling when the needle through vaginal wall; Grade 2: moderate pain, feel abdominal pain throughout the procedure with mild autonomic nervous dysfunction even when distracted, but patients still can tolerate; Grade 3: severe pain throughout the procedure with significant autonomic nervous dysfunction. Patients cannot tolerate and demand strongly to stop or suspend the surgeries. The patients' pain degrees were judged and recorded by a specific person from our study staff by single-blind method.

\subsection{Comparison of IVF-ET Outcomes}

The numbers of oocytes, fertilization rates, high-quality embryo rates, biochemical pregnancy rates were compared between the two groups. The biochemical pregnancy was defined as confirming blood $\beta$-HCG positive 14 days after embryo transfer.

\subsection{Statistical Analysis}

The Variance Analysis and $\chi^{2}$ test were performed for statistical analysis by using the SPSS 11.0. A P value less than 0.05 was considered statistically significant.

\section{Results}

\subsection{Comparison of the Perioperation Vital Signs}

In Table 1, it shows that the blood pressure, pulse and oxygen saturation are not statistically different before the oocyte retrieval operation between the two groups $(P>$ 0.05). During the operation, those differences were not significant yet $(P>0.05)$. But blood pressure and pulse in diclofenac suppository group reduced when compared with those in pethidine group $(P<0.001)$ after operation, except for oxygen saturation.

\subsection{Common Adverse Reactions during the Operations (Table 2)}

The incidences of nausea, vomiting and dizziness between the two groups were significantly different. The incidences in the diclofenac suppository group were relative lower $(P<0.001)$. No particular symptom rate between the two groups were also significantly different $(P$ $<0.001)$.

\subsection{Severe Adverse Events}

There were no death, emergency surgery and other major adverse events in the pethidine group during the six months of clinical study. In the diclofenac suppository group, no death has occurred. But there were three cases of abdominal bleeding with shock in it, which were required emergency laparotomies. The total blood loss was about $900 \mathrm{ml}, 1000 \mathrm{ml}$ and $2000 \mathrm{ml}$ respectively. The shock reasons were all ovarian bleeding. The time intervals between oocyte-retrievals and the sudden abdominal pain with shock were 8 hours, 20 hours and 9 hours respectively. No acute serious shock happened just after 
Table 1. Comparison of the perioperation vital signs between the two groups.

\begin{tabular}{|c|c|c|c|c|c|}
\hline & & Pethidine group & Diclofenac group & t Value & $P$ Value \\
\hline \multirow[t]{2}{*}{ Cases } & & 573 & 603 & & \\
\hline & Preoperation & $114.3 \pm 19.2$ & $112.9 \pm 16.7$ & 0.97 & 0.33 \\
\hline \multirow[t]{3}{*}{ Blood pressure (mmHg) } & Intraoperation & $110.7 \pm 22.7$ & $109.4 \pm 17.1$ & 1.11 & 0.25 \\
\hline & Postoperation & $101.2 \pm 17.7$ & $111.5 \pm 13.1$ & 11.38 & $<0.001$ \\
\hline & Preoperation & $85.4 \pm 8.9$ & $85.9 \pm 10.9$ & 0.86 & 0.39 \\
\hline \multirow[t]{3}{*}{ Pulse (beat/min) } & Intraoperation & $84.9 \pm 16.3$ & $85.1 \pm 11.8$ & 0.24 & 0.83 \\
\hline & Postoperation & $79.2 \pm 13.2$ & $83.4 \pm 10.1$ & 6.15 & $<0.001$ \\
\hline & Preoperation & $98.6 \pm 0.9$ & $98.5 \pm 1.0$ & 1.79 & 0.07 \\
\hline \multirow[t]{2}{*}{ Oxygen saturation (\%) } & Intraoperation & $98.5 \pm 1.0$ & $98.6 \pm 1.1$ & 0.24 & 0.09 \\
\hline & Postoperation & $98.6 \pm 0.9$ & $98.7 \pm 1.1$ & 1.70 & 0.07 \\
\hline
\end{tabular}

Table 2. Comparison of perioperation common adverse reactions (case).

\begin{tabular}{cccccc}
\hline & Case & Nausea & vomiting & Dizziness & No particular symptom \\
\hline Pethidine group & 573 & $161^{(\mathrm{a})}$ & $23^{(\mathrm{b})}$ & $48^{(\mathrm{c})}$ & $371^{(\mathrm{d})}$ \\
Diclofenac group & 603 & 73 & 6 & 21 & 519 \\
\hline
\end{tabular}

Compared with the study group, ${ }^{\text {(a) }} \chi^{2}=47.14, P<0.001$; ${ }^{(b)} \chi^{2}=11.13, P=0.001 ;{ }^{(c)} \chi^{2}=12.74, P<0.001 ;{ }^{\text {(d) }} \chi^{2}=72.58, P<0.001$.

operations.

\subsection{Comparison of the Pain Degree during the Oocyte Retrieval (Table 3)}

The pain degree was not statistically different between the two groups $(P=0.304)$. Most of the patients suffered mild or moderate pain during the operations.

\subsection{Comparison of IVF-ET Outcomes}

The differences of average oocyte, normal fertilization rate, number of available embryos rate and biochemical pregnancy rate were not statistically significant between the two groups $(P>0.05)$ (Table 4).

\section{Discussion}

In IVF-ET protocols, transvaginal puncture and oocyte aspiration guided by B-ultrasonic are invasive steps. Oocyte retrieval often brings fear and pain to some patients. Therefore the proper use of analgesic drugs is necessary. And it must ensure that the drug using in oocyte retrieval have no potential impact on the oocytes, embryos, and the future babies.

Pethidine analgesia is one of the most traditional analgesia methods in the IVF-ET oocyte retrieval, which is often used as a control of new analgesia methods. In clinic, pethidine is usually used together with diazepam in order to release the anxiety and fear of the patients. Dic-
Table 3. Comparison of the degree of pain between the two groups (case).

\begin{tabular}{|c|c|c|c|c|c|}
\hline & Case & Grade 0 & Grade 1 & Grade 2 & Grade 3 \\
\hline Pethidine group & 573 & 42 & 376 & 148 & 7 \\
\hline $\begin{array}{c}\text { Diclofenac } \\
\text { group }^{(a)}\end{array}$ & 603 & 54 & 412 & 132 & 5 \\
\hline
\end{tabular}

lofenac is an effective, opiate-sparing analgesic for acute pain, which is commonly used in pediatric surgical units [4]. So we speculated diclofenac should also be safe for IVF-ET. The main ingredient and its chemical name of diclofenac sodium suppository is 2-[(2,6-dichlorophenyl) amino]-Sodium Phenylacetate, a derivative of isobutyl diclofenac sodium. Its analgesic, anti-inflammatory and antipyretic effects are 2 to 2.5 times stronger than indomethacin, and 26 to 50 times than aspirin. Kailasam and Akande reported that using diclofenac suppository in IVF-ET had a good effect without obvious adverse effects [5,6]. The analgesic, anti-inflammatory effects of diclofenac work by inhibiting cyclooxygenase and reducing prostaglandin generation. And it also can inhibit the lipoxygenase, which reduces the production of leukotriene and bradykinin. In our study we used $50 \mathrm{mg}$ diclofenac suppository for analgesia and got a good effect. The drug has no adverse impact on the IVF-ET outcome, as the reports of Kailasam and Akande. But in both of 
Table 4. Comparison of the IVF-ET outcomes.

\begin{tabular}{cccccc}
\hline & Case & Oocyte $(n)$ & Normal fertilization rate $(\%)$ & Available embryo rate $(\%)$ & Pregnancy rate $(\%)$ \\
\hline Pethidine group & 573 & 6992 & $76.83(5372 / 6992)$ & $45.92(3211 / 6992)$ & $43.28(248 / 573)$ \\
Diclofenac group & 603 & 7831 & $74.01(5796 / 7831)$ & $44.86(3513 / 7831)$ & $42.12(254 / 603)$ \\
\hline
\end{tabular}

that two studies [5,6], diclofenac suppository was administered rectally immediately after the oocyte retrieval. In our study, diclofenac suppository was administered before the operation. And the outcome still indicated that the drug has no obvious adverse effects on IVF-ET outcomes even used before operation. The incidence of common adverse reactions induced by diclofenac suppository is lower than by pethidine. The probable reason is that the pethidine is a central analgesic, when intramuscular injecting it can cause autonomic nervous system disorder symptoms in some patients, such as dizziness, headache, nausea, vomiting, and so on. But diclofenac suppository is rectal administration, which is a local medication. So diclofenac suppository has slighter systemic symptom and seldom induce nervous and gastrointestinal disturbance. In addition, the result showed that the blood pressure and pulse were higher and recovered faster in diclofenac suppository group comparing with pethidine group. It indicates that diclofenac suppository group patients have more equability vital signs and less complication during oocyte retrieval. This requires further basic research to confirm. And the rectal administration by patients themselves can save the injection time of nurses and reduce the anesthetic management pressure. The above all may be the advantage of diclofenac suppository.

A study of Standing showed that the incidence of serious adverse reaction by using diclofenac suppository was $<0.8 \%$ [7]. In our study, 3 cases of severe abdominal bleeding happened after using diclofenac suppository, which needed emergency operations. And they took place more than 8 hours after the oocyte retrievals. Such cases in the control group did not appear, or so far rare. Reviewing the using of pethidine for oocyte retrieval analgesia in the near 5 years in our center, there was only one cases of severe abdominal bleeding. But she was a platelet dysfunction patient. Her pelvic vascular plexus was injured by puncture needle during the operation requiring emergency surgery to stop bleeding. Although it was still difficult to draw a conclusion because of the small cases in our study, it must pay attention to the relevance between diclofenac suppository and severe abdominal bleeding after oocyte retrieval. The diclofenac and aspirin are both non-steroidal anti-inflammatory and analgesic drugs. From the aspect of pharmacology, these drugs can inhibit the generation of prostaglandins, histamine and bradykinin with larger doses. But with small doses they can in- hibit prostaglandin cyclooxygenase (COX), reduce thromboxane A2 (TXA2) generation and inhibit platelet aggregation, which cause a bleeding tendency [8]. In this study, three cases of acute bleeding occurred after a long time of the oocyte retrieval, but not just after the puncture operations. Commonly when vascular were injured by the punctures, the bleeding should often occur immediately in normal coagulation patients. We speculate that the effective drug dose in rectum may decrease with time after treatment, and it lead to an anti-platelet effect and cause bleeding tendency. Reviewing the relative literature, we did not find the report about the severe abdominal bleeding after using diclofenac suppository for analgesic in oocyte retrieval yet.

In this study, we have not yet found an adverse effect on success rate in IVF-ET protocols with diclofenac suppository. However, some studies had found that prostaglandin cyclooxygenases (COXs), including COX-1 and $\mathrm{COX}-2$, play an important role in ovulation, implantation, and endometrial decidualization [9]. It may have an effect on the reproductive system in principle as diclofenac can inhibit the COXs. In this study, IVF-ET outcomes were similar between the two groups. The reason may be the short duration of drug action. But this was deserved further researches.

Reviewing the literature, we found that conscious sedation, propofol, nitrous oxide and acupuncture are used in oocyte retrieval analgesia of IVF-ET, which have different advantages and disadvantages [1,10]. Intravenous anesthesia is often considered the most comfortable. However, the studies of Wilhelm [11] and Boyers [12] had found adverse effects of intravenous anesthetics on the ooctyes and embryos. For this reason, Intravenous anesthesia is not the most popular method of anesthesia used in IVF protocol [13].

\section{Conclusion}

In summary, diclofenac suppository and pethidine both have good effects in oocyte retrieval analgesic. But the former has the advantages of ease use, relatively stable vital signs and fewer adverse reactions during operation. However, it must pay attention to the possibility of causing late-onset abdominal bleeding. A variety of oocyte retrieval analgesia methods are carried out, but different reproductive centers should choose the most suitable method based on different patients and centers. 


\section{Acknowledgements}

The work was supported by The National Natural Science Foundation of China (grant no. 81100470 and 81270750), The Young Teacher Training Project of Sun Yat-sen University, China (Grant No. 12ykpy24) and Natural Science Foundation of Guangdong Province, China (Grant No. S2012010009390).

\section{REFERENCES}

[1] N. F. Vlahos, I. Giannakikou, A. Vlachos and N. Vitoratos, "Analgesia and Anesthesia for Assisted Reproductive Technologies,” International Journal of Gynecology \& Obstetrics, Vol. 105, No. 3, 2009, pp. 201-205. doi:10.1016/j.ijgo.2009.01.017

[2] M. Vosoughin, S. Mohammadi and A. Dabbagh, "Intravenous Ketamine Compared with Diclofenac Suppository in Suppressing Acute Postoperative Pain in Women Undergoing Gynecologic Laparoscopy,” Journal of Anesthesia, Vol. 26, No. 5, 2012, pp. 732-737. doi:10.1007/s00540-012-1399-1

[3] S. Mitra, P. Khandelwal and A. Sehgal, "Diclofenac-Tramadol vs. Diclofenac-Acetaminophen Combinations for Pain Relief after Caesarean Section,” Acta Anaesthesiologica Scandinavica, Vol. 56, No. 6, 2012, pp. 706-711. doi:10.1111/j.1399-6576.2012.02663.x

[4] J. F. Standing, D. Tibboel, R. Korpela and K. T. Olkkola, "Diclofenac Pharmacokinetic Meta-Analysis and Dose Recommendations for Surgical Pain in Children Aged 1 12 Years," Pediatric Anesthesia, Vol. 21, No. 3, 2011, pp. 316-324. doi:10.1111/j.1460-9592.2010.03509.x

[5] C. Kailasam, L. P. Hunt, I. Ryder, I. Bhakri and U. D. Gordon, "Safety and Effectiveness of Diclofenac Sodium in Assisted Reproduction Treatment: A Randomized Prospective Double-Blind Study," Reproductive BioMedicine Online, Vol. 16, No. 5, 2008, pp. 724-729. doi:10.1016/S1472-6483(10)60488-9

[6] V. Akande, A. Garas and D. Cahill, "The Effect of Diclofenac and Paracetamol on Pregnancy and Implantation
Rates in Infertile Women Undergoing IVF Treatment," Journal of Obstetrics and Gynaecology, Vol. 26, No. 8, 2006, pp. 785-787. doi:10.1080/01443610600984578

[7] J. F. Standing, K. Ooi, S. Keady, R. F. Howard, I. Savage and I. C. Wong, "Prospective Observational Study of Adverse Drug Reactions to Diclofenac in Children," British Journal of Clinical Pharmacology, Vol. 68, No. 2, 2009, pp. 243-251. doi:10.1111/j.1365-2125.2009.03447.x

[8] L. C. Hamilton, J. A. Mitchell, A. M. Tomlinson and T. D. Warner, "Synergy between Cyclo-Oxygenase-2 Induction and Arachidic Acid Supply in Vivo: Consequences for Nonsteroidal Antiinflammatory Drug Efficacy,” FASEB Journal, Vol. 13, No. 2, 1999, pp. 245-251.

[9] H. Achache, A. Tsafrir, D. Prus, R. Reich and A. Revel, "Defective Endometrial Prostaglandin Synthesis Identified in Patients with Repeated Implantation Failure Undergoing in Vitro Fertilization," Fertility and Sterility, Vol. 94, No. 4, 2010, pp. 1271-1278. doi:10.1016/j.fertnstert.2009.07.1668

[10] A. Bokhari and B. J. Pollard, "Anaesthesia for Assisted Conception: A Survey of UK Practice," European Journal of Anaesthesiology, Vol. 16, No. 4, 1999, pp. 225230.

[11] W. Wilhelm, M. E. Hammadeh, P. F. White, T. Georg, R. Fleser and A. Biedler, "General Anesthesia versus Monitored Anesthesia Care with Remifentanil for Assisted Reproductive Technologies: Effect on Pregnancy Rate," Journal of Clinical Anesthesia, Vol. 14, No. 1, 2002, pp. 1-5. doi:10.1016/S0952-8180(01)00331-2

[12] S. P. Boyers, G. Lavy, J. B. Russell and A. H. DeCherney, "A Paired Analysis of in Vitro Fertilization and Cleavage Rates of First- versus Last-Recovered Preovulatory Human Oocytes Exposed to Varying Intervals of $100 \% \mathrm{CO}_{2}$ Pneumoperitoneum and General Anesthesia," Fertility and Sterility, Vol. 48, No. 6, 1987, pp. 969-974.

[13] P. O. Fiebai, A. A. Ogunmokun and R. A. Ajayi, "Experience with Conscious Sedation for Oocyte Retrieval in Nigeria," African Journal of Reproductive Health, Vol. 12, No. 1, 2008, pp. 30-34. 